www.nature.com/jhg

\title{
A Commentary on microRNA-141 confers resistance to cisplatin-induced apoptosis by targeting YAP1 in human esophageal squamous cell carcinoma
}

\author{
Naohiko Seki \\ Journal of Human Genetics (2011) 56, 339-340; doi:10.1038/jhg.2011.26; published online 10 March 2011
}

A lthough chemotherapy has been widely A used in treatment of cancer, drug resistance of cancer cells is a major clinical obstacle to curative effect and leads to poor prognosis of the patient. Many genetic (anticancer drug-induced random mutation of gene) and epigenetic (alteration of gene function without mutation) mechanisms have been reported to be the cause of resistance of cancer cells to chemotherapeutic agents. Although the anticancer drugresistance mechanisms have been extensively explored in protein code genes, the molecular mechanism has not yet been elucidated. ${ }^{1}$

Ever since the human genome-sequencing project era, studies with a new concept have indicated the existence and importance of another mechanism of gene function regulation by means of short-length non-coding RNA. For the conquest of drug resistance problem, understanding at the extensive molecule level, including non-coding RNA, is necessary. The microRNAs (miRNAs) are evolutionarily conserved small RNAs, which are single-stranded and consist of 19-23 nucleotides. They do not code for any protein or peptide; however, they regulate gene expression by inhibiting the protein translation process and/or degrading the respective target messenger RNA. ${ }^{2}$ Bioinformatic prediction indicates that miRNAs regulate more than $30 \%$ of the protein-coding genes. It is estimated that approximately 1000 miRNAs exist in the vertebrate genome. So far, 1048 human miRNAs have been registered

Dr N Seki is at the Department of Functional Genomics, Chiba University Graduate School of Medicine, 1-8-1 Inohana, Chuo-ku, Chiba, Japan.

E-mail: naoseki@faculty.chiba-u.jp at miRBase release 16.0 (http://microrna. sanger.ac.uk/). ${ }^{3}$

Recently, in the field of cancer research, accumulating evidence has reported that aberrant expression of miRNAs has a critical role in the initiation and progression of human cancer. ${ }^{4}$ In fact, recently an increasing number of articles have indicated the involvement of miRNAs in anti-cancer drug resistance. ${ }^{5}$ For instance, a recent study reported that miR-214 is upregulated in many ovarian tumors. miR-214 induces cell survival and cisplatin resistance by targeting the 3 -untranslated region of PTEN, which leads to downregulation of PTEN protein and activation of the Akt pathway. Interestingly, patients with recurrence of chemoresistance showed low miR-214 expression in the sensitive primary tumor and high miR-214 expression in tumors with recurring anti-drug resistance. This fact suggested that miRNA expression signature could be an effective tool to predict the therapeutic response to the anti-cancer drug treatment. ${ }^{6}$

In the Journal of Human Genetics Volume 56, Issue 4, Imanaka et al. ${ }^{7}$ suggest that miR-141 is involved in the development of cisplatin-resistant esophageal squamous cell carcinoma (ESCC) cell lines by directly targeting YAP1, a pro-apoptotic transcriptional factor. They selected miR-141 by comparing the miRNA expression profiles of the cisplatin-sensitive and resistant cell lines. Furthermore, when miR-141 was expressed ectopically in cisplatin-sensitive cell lines, cell viability after cisplatin treatment was significantly increased. From these results, they conclude that miR-141 has an important regulatory function in the development of cisplatin resistance in ESCC.
This experiments using ESCC cell lines were based on reliable data. As a matter of fact, validation in ESCC clinical specimens is necessary.

What is the function of miR-141 in ESCC? The function of miR-141 in human cancer is still controversial. miR-141 acts as a tumor suppressor in renal cell carcinoma and breast cancer cell lines, whereas it functions as an oncogene in nasopharyngeal carcinoma cells. ${ }^{8-10}$ Recent expression signatures in clinical specimens have indicated that miR-141 is downregulated in renal cell carcinoma and breast cancer, and upregulated in ovarian cancer. ${ }^{11}$ In nasopharyngeal carcinoma, bioinformatics and reporter assay confirmed that BRD3, PTEN and UBAP1 are the actual targets of miR-141. ${ }^{10}$ How about the miR141-regulated molecular network in ESCC carcinogenesis or development of cisplatin resistance? It is considerably difficult to completely understand the miRNA-regulated cancer network. Therefore, the study of the messenger RNA-miRNA network analysis remains a big genome puzzle to us.

1 Fojo, T. Multiple paths to a drug resistance phenotype: mutation, translocations, deletion and amplification of coding genes or promoter region, epigenetic changes and microRNAs. Drug Resist. Update 10, 59-67 (2007).

2 Bartel, D. P. MicroRNAs: genomics, biogenesis, mechanism, and function. Cell 116, 281-297 (2004).

3 Filipowics, W., Bhattacharyya, S. N. \& Sonenberg, N. Mechanisms of post-transcriptional regulation by microRNAs: are the answers in sight? Nat. Rev. Genet. 9, 102-114 (2008).

4 Calin, G. A. \& Crooce, C. M. MicroRNA signatures in human cancers. Nat. Rev. Cancer 6, 857-866 (2006).

5 Zheng, T., Wang, J., Chen, X. \& Liu, L. Role of microRNA in anticancer drug resistance. Int. J. Cancer 126, 2-10 (2010). 
6 Yang, H., Kong, W., He, H., Zhao, J. J., O'Donnell, J. D., Wang, J. et al. MicroRNA expression profiling in human ovarian cancer: miR-214 induces cell survival and cisplatin resistance by targeting PTEN. Cancer Res 68, 425-433 (2008).

7 Imanaka, Y., Tsuchiya, S., Sato, F., Shimada, Y., Shimizu, K., Tsujimoto, G. MicroRNA-141 confers resistance to cisplatin-induced apoptosis by targeting YAP1 in human esophageal squamous cell carcinoma. J. Hum. Genet. 56, 270-276 (2011).

8 Nakada, C., Matsuura, K., Tsukamoto, Y., Tanigawa, M., Yoshimoto, Y., Narimatsu, T. et al. Genome-wide microRNA expression profiling in renal cell carcinoma: significant down-regulation of miR-141 and miR-200c. J. Pathol. 216, 418-427 (2008).

9 Gregory, P. A., Bert, A. G., Paterson, E. L., Barry, S. C., Tsykin, A., Farshid, G. et al. The miR-200 family and miR-
205 regulate epithelial to mesenchymal transition by targeting ZEB1 and SIP1. Nat. Cell Biol. 10, 593-601 (2008).

10 Zhang, L., Deng, T., Li, X., Liu, H., Zhou, J., Wu, M. et al. microRNA-141 is involved in a nasopharyngeal carcinoma-related genes network. Carcinogenesis $\mathbf{3 1}$, 559-566 (2010).

11 Iorio, M. V., Visone, R., Di Leva, G., Petrocca, F., Casalini, P., Taccioli, C. et al. MicroRNA signatures in human ovarian cancer. Cancer Res. 67, 8699-8707 (2007). 